\title{
El aporte de las cooperativas a las políticas públicas y el territorio: un análisis de las mediciones estadísticas en Argentina
}

\section{Cooperatives' contribution to public policies and territory: Analysis of Argentinian statistical measurements'}

\author{
María Eugenia Castelao-Caruana ${ }^{1} \bowtie$
}

${ }^{1}$ Investigadora asistente del Consejo Nacional de Investigaciones Científicas y Técnicas, profesora de la Facultad de Ciencias Económicas de la Universidad de Buenos Aires.

Correo electrónico: eugecastelao@gmail.com

Recibido: 30 de julio del 2017 Aprobado: 15 de diciembre del 2017 Disponible en línea: 1 de enero del 2018

Cómo citar este artículo: Castelao-Caruana, M. E. (2018). El aporte de las cooperativas a las políticas públicas y el territorio. Un análisis de las mediciones estadísticas en Argentina, Cooperativismo \& Desarrollo, 113(26). doi: https://doi.org/10.16925/ co.v26i113.2190

\section{Resumen}

Introducción: en las últimas décadas, los poderes públicos nacionales y los organismos internacionales han incrementado su interés por la forma de organización cooperativa, y reconocen su capacidad de contribuir al abordaje de problemas sociales, económicos y ambientales.

Metodología: la metodología de esta investigación se basa en el modelo analítico de políticas púbicas que propone comprender la lógica que encierra la acción de las instituciones políticas y de los actores involucrados en las intervenciones públicas desde el ángulo de su contribución a la solución de los problemas colectivos.

Resultados: el trabajo estudia el papel de las cooperativas en las políticas públicas a partir del análisis de las intervenciones públicas de alcance nacional, vigentes en Argentina en el periodo 2003-2015. Por otra parte, se identifican y analizan las distintas fuentes de información disponibles en el país que podrían dar cuenta de las acciones que realizan estas entidades.

Conclusiones: se plantea la necesidad de registrar y medir el peso económico y social de las cooperativas y el impacto de su participación en las políticas públicas a fin de producir un mejor conocimiento sobre la realidad del sector, fortalecer su imagen positiva y difundir esta forma de organización.

Palabras clave: balance social cooperativo, cooperativas, evaluación de impacto, indicadores económicos, medidas estadísticas, políticas públicas. 


\title{
Cooperatives' contribution to public policies and territory: Analysis of Argentinian statistical measurements'
}

\begin{abstract}
Introduction: In recent decades, national public authorities and international organizations have become growingly interested in cooperative organization and recognize its possibility to contribute social, economic and environmental problems approach.

Methodology: The methodology of this research is based on a public policies analytical model that aims to understand the logic that underlies political institutions and actors involved in public interventions' actions with respect to their contribution to solve collective problems.

Results: The paper studies the role of cooperatives in public policies based on the analysis of public interventions of national scope given in Argentina from 2003 to 2015. Also, the different sources of information available in the country that could disclose the actions carried out by these entities are identified and analyzed.

Conclusions: There is a need to register and measure cooperatives' economic and social importance and the impact of their participation in public policies in order to attain better understanding of the sector's reality, strengthen its positive image and publicize this form of organization.

Keywords: cooperative social balance, cooperatives, impact evaluation, economic indicators, statistical measures, public policies.

\section{A contribuição das cooperativas para as políticas públicas e o território: uma análise das medidas estatísticas na Argentina}

\section{Resumo}

Introdução: nas últimas décadas, as autoridades públicas nacionais e as organizações internacionais aumentaram o seu interesse pela forma de organização cooperativa, e reconheceram a sua capacidade de contribuir para o tratamento de problemas sociais, económicos e ambientais.

Metodologia: a metodologia da presente investigação baseia-se no modelo analítico de políticas públicas que propõe compreender a lógica em torno à ação das instituições políticas e os atores envolvidos em intervenções públicas, a partir do ângulo de sua contribuição para a solução de problemas coletivos.

Resultados: 0 artigo estuda o papel das cooperativas nas políticas públicas a partir da análise de intervenções públicas de abrangência nacional, vigentes na Argentina no período 2003-2015. Por outro lado, identificam-se e analisam-se as diferentes fontes de informação disponíveis no país, que poderiam explicar as ações realizadas por essas entidades.

Conclusões: expõe-se a necessidade de registrar e medir o peso econômico e social das cooperativas e o impacto de sua participação nas políticas públicas, a fim de melhor compreender a realidade do setor, fortalecer sua imagem positiva e disseminar essa forma de organização.

Palavras-chave: equilíbrio social cooperativo, cooperativas, avaliação de impacto, indicadores econômicos, medidas estatísticas, políticas públicas. 


\section{Introducción}

En las últimas décadas, los Estados nacionales y los organismos internacionales han diseñado e implementado múltiples políticas con referencias a la forma cooperativa, lo cual ha contribuido en algunos casos a una mayor democratización de la economía y de la sociedad (Vaillancourt, 2009), y en otros a paliar los desequilibrios y los ajustes del sistema. En la práctica, sin embargo, estas políticas pocas veces conciben a las cooperativas $-\mathrm{y}$ al movimiento cooperativo- como un actor central de sus acciones (Chaves, 2012). Se trata de políticas con enfoques y métodos heterogéneos que responden al entorno político, cultural e institucional de cada país, así como a su definición de la relación Estado-sociedad y de la articulación entre la esfera económica (considerada productiva) y social (asociada a la redistribución). Además, son políticas experimentales que enfrentan grandes desafíos para ser eficaces, dada su fragilidad y vulnerabilidad institucional en relación con las coyunturas políticas (Chaves y Demoustier, 2013; Organización Internacional del Trabajo-oit, 2011).

En este contexto, analizar la participación de las cooperativas en la etapa de implementación de las políticas públicas, evaluar su capacidad de gestionar los recursos públicos y comprender su peso y papel en la economía y en la sociedad, son acciones claves en el propósito de evaluar las intervenciones públicas y facilitar la toma de decisiones de actores públicos y privados. Asimismo, el estudio del impacto de las cooperativas en sus territorios, así como su evolución económica en relación con otras formas de organización empresarial, permite debatir con fundamentos el papel que otorgan las intervenciones públicas a las cooperativas como fuente de crecimiento económico y desarrollo comunitario. En este sentido, la producción de estadísticas e indicadores sobre las cooperativas debe orientarse a cuantificar su peso económico y social, fortalecer su imagen positiva, producir conocimiento sobre el sector y difundir esta forma de organización (Artis, Bouchard y Rousseliere, 2015).

En este marco, este trabajo identifica, por una parte, la manera en que las cooperativas participan en las políticas públicas de alcance nacional vigentes en Argentina en el periodo 2003-2015, y, por otra, analiza las mediciones utilizadas para registrar el aporte y el desempeño de estas organizaciones, en el marco de distintas políticas públicas y en la relación con sus asociados. El artículo se organiza de la siguiente manera: luego de presentar la metodología de trabajo, analiza los tipos de políticas públicas que han involucrado a las cooperativas, sus formas de participación, sus desafíos y sus oportunidades; en la sección siguiente, ahonda en el alcance de las fuentes de información nacionales, públicas y privadas, que hacen referencia a las cooperativas, y su utilidad para evaluar los recursos, los productos, los resultados y los impactos de estas entidades en la sociedad. Por último, se presentan algunas reflexiones acerca de la participación de las cooperativas en las políticas públicas, y las restricciones y posibilidades que presentan las fuentes de información actualmente disponibles en el país para fortalecer la imagen, el conocimiento y la participación del movimiento cooperativo.

\section{Metodología}

La metodología de esta investigación se basa en el modelo analítico de políticas púbicas propuesto por Subirats, Knoepfel, Larrue y Varone (2008), el cual propone comprender la lógica que encierra la acción de las instituciones políticas y de los actores involucrados en las intervenciones públicas desde el ángulo de su contribución a la solución de los problemas colectivos, y el cual da cuenta, a su vez, de la heterogeneidad y la fragmentación que caracteriza a los actores públicos y privados.

En este marco conceptual, la investigación analizó la formulación e implementación de las políticas públicas nacionales con referencia a la forma de organización cooperativa vigente en Argentina durante el periodo 2003-2015. La formulación de una política contempla un programa de actuación y de acuerdos de actuación político-administrativos. El primero constituye la fuente de legitimidad primaria de una política y lo conforman las bases jurídicas que sustentan sus objetivos, sus elementos evaluativos, sus operativos, sus procedimentales y sus recursos asignados. Los acuerdos de actuación, por su parte, hacen referencia a las características de los actores públicos y privados, la distribución de competencias y responsabilidades entre estos y los recursos de los que disponen los actores locales para incidir sobre las formas de implementación previstas en sus territorios (Subirats et al., 2008).

La etapa de implementación contempla el conjunto de productos finales de los programas que se destinan de manera individual a las personas que 
forman parte de los grupos-objetivo previstos, los niveles territoriales en los que se concretan estos productos y la capacidad de incidencia de los actores locales y de las cooperativas, en particular, en las formas de implementación de estos programas.

Este análisis permite, por una parte, clasificar las políticas públicas en atención a sus objetivos en materia de desarrollo económico y social, y según el papel de las cooperativas en la formulación e implementación de estas políticas públicas. La descripción y los resultados de este análisis se encuentran desarrollados en el capítulo 3 de la tesis de doctorado La economía social y solidaria en las políticas públicas nacionales y su incidencia en el territorio: alcance y perspectivas (Castelao Caruana, 2013). Por otra parte, con base en los programas de actuación, se analizaron las medidas utilizadas por estos programas para registrar los insumos, los productos, los resultados y los impactos de estas políticas públicas.

Para la realización de este trabajo se recurrió a fuentes de datos primarios y secundarios, las cuales se utilizaron de forma complementaria. Las fuentes de datos primarios contribuyeron a construir un enfoque más amplio y dinámico de los programas y los acuerdos de actuación y, en particular, de la incidencia de los actores locales y de las cooperativas sobre las formas de implementación de estos programas. Se aplicaron entrevistas a informantes claves del Gobierno nacional, de gobiernos municipales y a referentes de diversas cooperativas. Las entrevistas realizadas se basaron en una guía de preguntas adaptada en función del rol y las actividades de cada interlocutor.

Como fuente de datos secundarios se recurrió a información elaborada por organismos públicos y privados: fuentes documentales referidas a la formulación e implementación de las políticas públicas y a la participación de los distintos actores en estas etapas; normas y regulaciones que institucionalizan la actividad de las cooperativas y que definen los programas y los acuerdos de actuación político-administrativos de las políticas públicas bajo análisis; y estadísticas públicas y privadas que hacen mención a las cooperativas.

\section{La participación de las cooperativas en las políticas públicas}

Luego de la pronunciada crisis económica y social que vivió Argentina a fines del 2001, el Gobierno nacional comenzó a aplicar en el 2003 una serie de reformas económicas y políticas que derivaron en una mayor participación del Estado nacional en la regulación de la economía, la redistribución de la riqueza y la atención de las necesidades sociales. Además, el Gobierno nacional estableció una nueva relación con los movimientos sociales al incorporar parte de sus reclamos en la agenda estatal, no sin discrepancias respecto a la concepción de los problemas y la definición de sus causas (Cortés, 2008).

De esta forma, la exclusión y su incidencia en el desempleo y la pobreza cobraron importancia en la agenda política y en el discurso del Gobierno nacional, en el marco de un enfoque redistributivo (centrado en los límites que impone la pobreza material a la participación social y el ejercicio de los derechos ciudadanos) y de integración social (el cual reafirma la centralidad del empleo como mecanismo de integración económica y social). Las prioridades de esta agenda gubernamental y sus tensiones sociales implícitas se plasmaron a lo largo del periodo 2003-2015 en políticas públicas con un enfoque socioproductivo que incorporaron en su formulación diversas formas de organización identificadas por el Estado con la economía social, entre ellas las cooperativas. Vale la pena aclarar que no existe información fehaciente sobre el número de asociados a cooperativas a nivel nacional, ni sobre el PBI que generan las entidades. A modo de ejemplo, el censo nacional realizado por el Inaes afirma que en la provincia de Santa Fe existen 2755751 asociados, mientras que la población total de la provincia (incluidos menores) es de 3194 537. Es posible que la metodología del censo no pudo evitar el doble conteo de personas asociadas a más de una cooperativa, práctica común en localidades de provincias como Santa Fe.

En el 2001, el cooperativismo en el país estaba formado por 16059 entidades, de las cuales 6686 (42\%) eran cooperativas de trabajo, 3060 (19\%) de vivienda y construcción, y 2236 (14\%) agropecuarias. Unos 13 años más tarde, el número de entidades registradas en el Instituto Nacional de Asociativismo y Economía Social (Inaes) había crecido en un $79 \%$, debido al aumento en el número de cooperativas de trabajo registradas a través de políticas sociales. Por otra parte, el número de cooperativas agropecuarias, de seguros, vivienda y, en menor grado, de consumo, crédito y provisión, continuó su disminución como consecuencia de 
procesos estructurales activos desde la década de 1960. A pesar de esta tendencia, estas cooperativas representaban el 1,4\% de los puestos de trabajo registrados por las empresas privadas del país (Vuotto, 2014). Los integrantes de cooperativas de trabajo, por otra parte, sumaban alrededor de 177000 personas en el 2007 (112 112 asociados a cooperativas de trabajo tradicionales y 65456 integrantes de cooperativas de trabajo creadas en el marco de políticas sociales) (Inaes, 2008). No obstante, en el 2011, 180000 cooperativistas efectuaban actividades de saneamiento, equipamiento e infraestructura urbana en el marco del Programa Social con Trabajo (Vuotto, 2014).

La intensidad y el modo en que las cooperativas se involucran en la resolución de los problemas públicos depende tanto de su reconocimiento político como de la interacción con los actores públicos implicados en el surgimiento, la formulación y la implementación de las políticas públicas. La participación de las cooperativas en las políticas públicas se analiza a partir de dos tipologías. La primera se basa en la orientación en materia de desarrollo económico y social que adoptan las políticas públicas y ha sido desarrollada por diversos autores (Amyot, Downing y Tremblay, 2010; Tremblay, 2010) que distinguen cuatro categorías: políticas de reconocimiento político, políticas sectoriales, políticas sociales, y políticas con enfoque territorial. La segunda tipología, desarrollada a partir del análisis de las políticas públicas bajo estudio, se centra en el modo en que se involucran las cooperativas en la resolución de los problemas públicos en el papel de promotoras, beneficiarias o instrumentos de organización. Los siguientes párrafos describen el alcance de cada una de estas tipologías y su aplicación en las políticas públicas bajo análisis.

\section{Una tipología de políticas públicas asociadas al cooperativismo ${ }^{1}$}

Las medidas de reconocimiento político registran a las cooperativas como una forma de organización productiva viable, autónoma del Estado y representativa de los intereses de sus asociados. Su existencia es una condición necesaria para que el cooperativismo construya relaciones simétricas

1 Este apartado resume la descripción y el análisis de las políticas públicas nacionales desarrollada en el Capítulo 3 de la tesis de doctorado (Castelao Caruana, 2013). con el Estado en distintos espacios de interacción. De esta forma, las cooperativas adquieren entidad para participar en la elaboración de políticas públicas, plantear proyectos y metas de desarrollo que involucren a diferentes niveles de gobierno y perduren a lo largo de distintas gestiones gubernamentales.

Algunas medidas institucionales que reflejan este tipo de política son las destinadas a eliminar obstáculos jurídicos para el desarrollo del cooperativismo. De acuerdo con Chaves (2012) se trata de normas que:

- Definen su forma jurídica como actor privado.

- Reconocen la capacidad de operar de las cooperativas en todo sector de actividad económica.

- Reconocen a las cooperativas como un actor político, como interlocutor en la elaboración y en la ejecución de políticas públicas.

- Otorgan impulso de organismos públicos de fomento de las cooperativas.

En Argentina, la Ley 20.337 de 1973 reglamenta la creación, el funcionamiento, la disolución y la liquidación de las cooperativas. El movimiento cooperativo ha planteado la necesidad de modificar esta ley, al propiciar la sanción de leyes especiales que contemplen la realidad económica actual y las peculiaridades de las diversas formas cooperativas. Sin embargo, los principales problemas jurídicos del sector no provienen de las limitaciones de la ley, sino de la existencia de otras normas generales que discriminan a las entidades cooperativas o no consideran su naturaleza particular, especialmente en las áreas de prestación de servicios públicos, de telefonía celular y de servicios financieros, entre otros (Instituto Movilizador de Fondos CooperativosIMFC, 2008), lo cual limita su posibilidad de actuar en distintos sectores económicos.

Otra política de reconocimiento es la Ley de Monotributo Social (Ley 25.865 de 2004) que establece un régimen tributario para los asociados a cooperativas de trabajo en situación de vulnerabilidad. $^{2}$ Inicialmente, su objetivo fue regularizar la situación laboral de quienes, a través de programas públicos, constituían cooperativas de trabajo

2 Al abonar mensualmente el 50\% del componente social, este régimen permite a los inscriptos emitir factura oficial, acceder a una obra social de libre elección y computar el periodo de inscripción como años de aporte jubilatorio. 
para la realización de tareas provistas por el Estado. Posteriormente, los beneficios de este régimen tributario se extendieron a todos los integrantes de cooperativas de trabajo y emprendimientos autogestionados en situación de vulnerabilidad.

La Ley Nacional de Promoción del Microcrédito para el Desarrollo de la Economía Social (Ley 26.117 de 2006), por otra parte, tiene como objetivo ampliar el acceso al crédito de pequeños emprendimientos productivos. Esta ley promueve un modelo de gestión asociada que combina la articulación pública-privada y la complementariedad de las acciones del Estado en sus diferentes jurisdicciones. Concibe a las cooperativas - entre otras entidades-como agentes coejecutores de esta política pública, y reconoce su condición de interlocutor en los territorios y la necesidad de fortalecer el capital social en ellos.

Las políticas sectoriales otorgan preferencia a las cooperativas en el abordaje de problemas públicos tales como la escasez de viviendas y la restricción de acceso al crédito, y en la promoción de determinados sectores de actividad que contribuyen a la inclusión y el desarrollo local, como, por ejemplo, los servicios personales (guarderías, cuidado de personas mayores) y de enseñanza o de salud, el desarrollo de nuevas tecnologías, el turismo comunitario y la cultura, entre otras. Estas políticas también contemplan acciones específicas cuyo propósito es mejorar la función empresarial y social de las entidades mediante instrumentos financieros, impositivos, formativos y tecnológicos (Chaves, 2012).

En Argentina, las políticas sectoriales dirigidas a otorgar preferencia a las empresas cooperativas han intervenido, principalmente, en la construcción de soluciones habitacionales y en obras de saneamiento. El Estado abordó estos problemas públicos mediante la contratación de empresas privadas de capital, por un lado, y la conformación de cooperativas de trabajo por otro, en el marco de políticas de inclusión social (Programa Federal de Integración Socio-comunitaria por Cooperativas-PFISC). Sin embargo, el tratamiento que otorgó el Estado a las cooperativas no fue preferencial y dicha contratación no formó parte de una estrategia compartida entre el Estado y el sector cooperativo de vivienda o de trabajo.

Los programas sectoriales implementados por organismos descentralizados del Gobierno nacional han sido más efectivos en sus resultados. Estos programas han promovido la incorporación de prácticas de gestión eficientes entre las cooperativas mediante instrumentos financieros, impositivos y tecnológicos adecuados a sus características particulares, transversales al resto de las políticas públicas e independientes de la situación socioeconómica de sus integrantes. El objetivo de este tipo de política es que las cooperativas compitan en el mercado sin comprometer sus objetivos sociales y sin requerir un apoyo sostenido del Estado. Entre las acciones públicas desarrolladas en esta área de intervención se han destacado algunos programas del Instituto Nacional de Tecnología Industrial (INTI) y del Inaes, organismos descentralizados del Estado nacional.

A diferencia de las políticas anteriores, las políticas sociales favorecen a un determinado segmento de la población en condiciones de vulnerabilidad por la falta de empleo, ingresos, formación o acceso a bienes y servicios. Los programas sociales que incorporan a las cooperativas de trabajo en su etapa de diseño y formulación reconocen algunas de las prácticas y principios que las guían - gestión participativa, propiedad colectiva, cooperación y solidaridad en las relaciones externas-. Estas políticas públicas buscan adoptar un enfoque socioproductivo distante de la lógica asistencial y descentralizada que privilegiaron las políticas sociales de la década de los noventa (Vinocur y Halperin, 2004).

Así, mientras algunas políticas sociales establecen la constitución de cooperativas de trabajo como condición para acceder a beneficios sociales (Programa Ingreso con Trabajo-Prist), otras incorporan a las cooperativas como agentes intermediarios en la etapa de implementación de las políticas públicas, como es el caso del Programa de Microcrédito creado a partir de la Ley Nacional de Promoción del Microcrédito para el Desarrollo de la Economía Social mencionada.

Por último, las políticas con enfoque territorial acompañan los procesos productivos y sociales que se desarrollan en los territorios - y no exclusivamente en las unidades productivas que los componen- mediante instrumentos amplios, flexibles e integrados. Son medidas amplias porque pueden intervenir sobre diversas dimensiones (sociales, económicas, institucionales); flexibles, porque se adaptan a las necesidades específicas de cada proyecto o comunidad; e integradas, porque son gestionadas por una misma área de administración pública o por áreas diferentes con mecanismos de coordinación efectivos. 
Estas políticas integran objetivos económicos, sociales y ambientales, y se orientan a promover proyectos que fortalecen las capacidades del territorio y potencian su desarrollo. El enfoque territorial se plantea como necesario tanto en las políticas productivas (Ropert, 2009) como en aquellas dirigidas a abordar el desempleo (Casanova, 2004) y la pobreza (Klein et al., 2010).

Respecto a las normas que dan origen a estos programas, debo decir que no hay normativa pública que los sustente. El INTI es un organismo descentralizado y las decisiones administrativas que dan origen a sus programas, si existen, no son públicas. La Red Argentina es una acción sistemática impulsada por el sector público, pero desarrollada en el ámbito privado, por lo que tampoco tiene una norma que lo sustente.

Ejemplos de políticas diseñadas con enfoque territorial en Argentina son el programa Cadena de Valor Artesanal - que formó parte del Programa de Extensión del INTI-, y la Red Argentina de Turismo Rural Comunitario, una intervención impulsada en el marco del Programa de Desarrollo de Espacios Turísticos del Ministerio de Turismo de la Nación, pero formulada e implementada en el ámbito privado. Mediante instrumentos de política propios o al facilitar el acceso a otros programas y recursos, ambas acciones contribuyen a la construcción y el fortalecimiento de los emprendimientos (algunos de ellos cooperativas) como parte de un entramado productivo que, directa o indirectamente, promueve el desarrollo de los territorios. En una situación intermedia se encuentran el Plan Manos a la Obra (Resolución spsydH 1.375/2004) y el programa de Promoción del Microcrédito (Ley 26.117 de 2006), cuya implementación descansa en la articulación de las cooperativas como entidades intermediarias locales y la población beneficiaría. Ambos programas proponen la redefinición de las responsabilidades frente a los problemas de desempleo y exclusión social al ubicar a las entidades intermediarias como promotoras de experiencias productivas y a la población beneficiaria como sujeto activo.

\section{Formas de participación de las cooperativas en las políticas públicas}

En el marco de las distintas categorías de políticas públicas descritas, las cooperativas pueden adoptar distintos roles según las funciones que adopta su participación. Cada uno de estos roles ofrece oportunidades que el movimiento cooperativo puede potenciar y desafíos que debe atender (véase la tabla 1).

Tabla 1

Función de las cooperativas en las políticas públicas

\begin{tabular}{lll}
\hline \multicolumn{1}{c}{ Rol } & \multicolumn{1}{c}{ Oportunidades } & \multicolumn{1}{c}{ Desafíos } \\
\hline Promotora & $\begin{array}{l}\text { Participación en la } \\
\text { coproducción de } \\
\text { la política pública }\end{array}$ & $\begin{array}{l}\text { Tercerización del } \\
\text { rol del Estado. }\end{array}$ \\
Beneficiaria & $\begin{array}{l}\text { Fortalecimiento } \\
\text { económico e } \\
\text { institucional }\end{array}$ & $\begin{array}{l}\text { Escasa autonomía } \\
\text { económica (y polí- } \\
\text { tica) del Estado. }\end{array}$ \\
$\begin{array}{l}\text { Instrumento de } \\
\text { organización }\end{array}$ & $\begin{array}{l}\text { Difusión del mo- } \\
\text { delo cooperativo }\end{array}$ & $\begin{array}{l}\text { Desvío de los } \\
\text { principios coope- } \\
\text { rativos. }\end{array}$ \\
\hline
\end{tabular}

Nota. Elaboración propia.

Como promotoras, las cooperativas participan en la etapa de implementación de las políticas públicas. Estas son el marco de acción a partir del cual las cooperativas involucradas en su implementación construyen sus propias estrategias de intervención en los territorios. Los recursos de los que disponen estas entidades, así como su producción, gestión, explotación, combinación e incluso su intercambio, influyen en el diseño, el proceso y el resultado de estas intervenciones y, particularmente, en la manera en que la organización se inscribe en el proceso de desarrollo de su territorio.

Las cooperativas son organizaciones creadas para dar respuesta a las necesidades de sus miembros en el marco de procesos organizacionales democráticos, de manera que proponen soluciones para los problemas colectivos basadas en la articulación estado-mercado-sociedad, por la cual se supera el modelo tradicional de intervención pública (Estado-mercado). Sin embargo, cuando la participación implica la transferencia de las responsabilidades del Estado hacia las cooperativas -en algunos casos mediada por intereses político-clientelares, pero siempre caracterizada por el escaso apoyo en términos de recursos humanos, monetarios y políticos (Pestoff, 2006)—, este ámbito de participación se transforma en un espacio de tercerización (Rouillard, 2006). De esta forma, 
la relación entre el Gobierno y estas entidades se reduce a una subcontratación mercantil que impide construir y desarrollar sinergia entre organizaciones públicas y privadas. En estos casos, las cooperativas en su papel de promotoras, movilizan recursos propios (monetarios, humanos, políticos, etc.) a fin de potenciar las posibilidades de éxito de los beneficiarios.

El Programa de Microcrédito, derivado de la Ley Nacional de Promoción del Microcrédito, por ejemplo, ha otorgado a las cooperativas el rol de promotoras con vistas a ampliar el acceso al crédito productivo y disminuir la vulnerabilidad o la exclusión social de sectores de la población.

Las cooperativas pueden ser también destinatarias y beneficiarias de las políticas públicas, especialmente en el marco de políticas sectoriales como las que han implementado el INTI y el Inaes. El fortalecimiento económico e institucional del sector y su consolidación como movimiento orgánico requiere la reasignación de los recursos públicos hacia políticas que reconozcan la especificidad del cooperativismo y su capacidad de actuar en ciertos mercados, fortaleciendo su interacción con el mercado, la tecnología y el propio Estado (Pochmann, 2007; Singer, 2007). Las estructuras de apoyo al sector y sus políticas específicas son claves en este proceso, en especial cuando extienden sus funciones más allá de la reglamentación, la fiscalización y la estadística, con el fin de desarrollar tecnologías y ofrecer servicios de financiación, asistencia técnica y formación adaptados a la realidad y a la estructura del sector (Spear, 2000).

Las políticas sectoriales pueden fortalecer la actividad económica de las cooperativas y sus procesos de organización productiva e institucional, pero la transferencia de recursos desde el Estado y la incidencia de sus técnicos en las esferas de gestión también pueden plantear dudas acerca de su autonomía económica y, consecuentemente, de su independencia política.

Algunas políticas públicas incorporan a las cooperativas en su formulación como forma jurídica adecuada para organizar a la población beneficiaria. Este tipo de participación instrumental representa una oportunidad para la difusión del cooperativismo entre distintos sectores al promover su crecimiento y la diversificación de voces, intereses y realidades al interior del movimiento.

Sin embargo, las cooperativas creadas en el marco de estos programas, por lo general, no son resultado de la unión voluntaria de sus miembros, sino una decisión del Estado. Es así como, para muchos beneficiarios, estas entidades constituyen meramente un medio para resolver sus problemas individuales de empleo, vivienda y acceso al crédito, entre otros. Esta lógica de promoción y funcionamiento conduce a una visión de corto plazo que valoriza la importancia de los resultados inmediatos y dificulta generar normas compartidas relativas al carácter asociativo de las entidades. Aunque el tejido de lazos y relaciones sociales que construyen las cooperativas puede resultar estabilizador y reproductivo, su creación con base en la necesidad puede generar también una pérdida importante de autonomía.

Adicionalmente, el financiamiento de las cooperativas creadas en el marco de una política pública permite a las autoridades, de manera más o menos marcada, imponer sus instrumentos de control. Además, puede animar a las organizaciones a seguir una simple "lógica del subsidio" que, en general, tiende a limitar su potencialidad y condicionar su autonomía e iniciativa. En este contexto, es complejo para estas organizaciones preservar sus principios, al no contar con capacidad para autofinanciar su actividad - aunque solo sea parcialmente-; a la inversa, también se presentan límites cuando el objetivo de autofinanciamiento las conduce a adoptar prácticas exclusivamente mercantiles. Este ha sido el rol de las cooperativas en algunas de las políticas sociales y sectoriales implementadas en los últimos años por el Estado.

\section{El alcance de las mediciones sobre cooperativas}

\section{Elementos estratégicos de una política pública.}

La fase de formulación de una política supone la definición de un modelo causal y el diseño de un programa de actuación político-administrativo (Subirats et al., 2008) que incluye la identificación de sus elementos estratégicos: insumos, productos, resultados e impactos u objetivos. Este programa describe, además, las relaciones de causa y efecto esperadas entre estos elementos y los indicadores de riesgo que pueden influir en el éxito o en el fracaso del programa (Guanziroli, Buainain y de Sousa Filho, 2007). 
Los insumos de una política se refieren al conjunto de recursos ${ }^{3}$ que movilizan los actores para, mediante distintas acciones o actividades, generar productos. Los productos son logros concretos y observables de un programa o proyecto (manuales, libros, talleres de formación, etc.). En otras palabras, son los bienes y servicios proporcionados a la población beneficiaria o a los grupos intermediarios para la generación de resultados. Aunque se pueden confundir con los productos, los resultados son una evaluación cualitativa y cuantitativa de la entrega y la calidad de los productos (Guanziroli et al., 2007). A cada objetivo planteado y descrito en el programa de actuación le corresponderá al menos un producto y resultado. Sin embargo, los programas aspiran a generar impactos más allá de sus resultados, los cuales suelen ser la razón primordial de la política pública.

\section{Mediciones públicas y privadas.}

Los organismos públicos nacionales ofrecen información sistemática sobre los insumos aplicados, las actividades desarrolladas y los productos alcanzados por cada intervención pública, mediante documentos trimestrales como, por ejemplo, los informes de la Contaduría General de la Nación de la Cuenta de Inversión, los Informes de Ejecución Físico-Financiera del Presupuesto de la Administración Nacional, y documentos de periodicidad anual como las "Memorias del estado de la Nación". Estas fuentes secundarias brindan información sobre el avance material de los programas y proyectos en relación con las metas establecidas, y evalúan periódicamente los recursos destinados al programa, las acciones encaradas y los productos y resultados alcanzados (véase la tabla 2).
Otros documentos públicos relevantes para conocer los elementos estratégicos de una política pública son los informes de la Sindicatura General de la Nación y los informes de resultado o evaluación encargados por los organismos públicos que implementan la política pública en cuestión. Sin embargo, la información provista en estos documentos en particular no se presenta de manera periódica ni sistemática, y los informes que incluyen algún tipo de evaluación de los programas ${ }^{4}$ han tenido escasa visibilidad pública, de manera que es, por consiguiente, limitada su accesibilidad. Es importante notar, sin embargo, que la mayoría de las políticas públicas en Argentina no contemplan modalidades de evaluación de impacto - a posteriori de la ejecución de los programas- como parte de su estrategia.

Aunque es habitual que los actores públicos se centren en medir e informar sobre los recursos y productos inmediatos de las políticas públicas (Gertler, Martínez, Premand, Rawlings y Vermeersch, 2017), estos datos no alcanzan para mejorar las intervenciones y su gestión, promover procesos de aprendizaje organizacional, estimar el coste social de la política pública o evaluar el logro de sus objetivos (sus resultados e impactos). En otras palabras, al centrarse en la gestión del Estado, estos documentos, por un lado, desconocen los recursos que las cooperativas - como beneficiarias o como promotoras - movilizan en las etapas de formulación e implementación de las políticas públicas. Estos insumos y su gestión son claves para entender el alcance de estos programas, su verdadero costo social y la incidencia de las cooperativas en sus resultados e impactos.

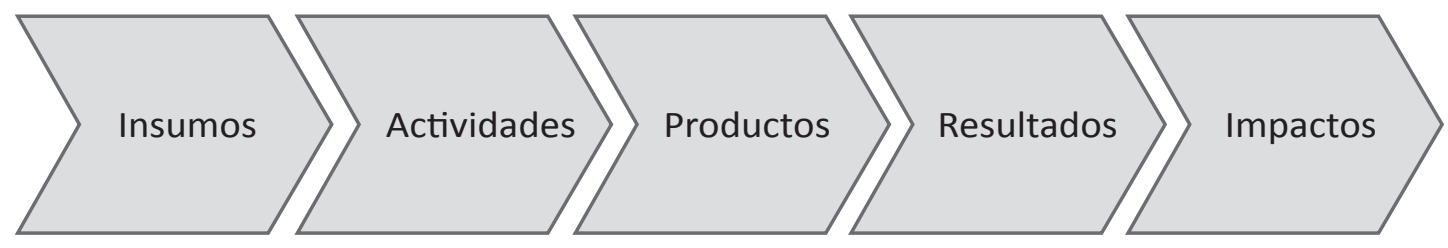

Figura 1. Elementos estratégicos de una política pública. Elaboración propia.

3 Al hablar de la movilización de recursos se hace referencia a la producción, la combinación y la sustitución de recursos económicos (dinero), humanos (personal), jurídicos, cognitivos (información), interactivos (organización), políticos (apoyo de la mayoría), patrimoniales (infraestructura), de confianza (consenso, solidaridad), y cronológicos (tiempo).

4 Los programas "Plan Manos a la Obra" y "Prist" aplicaron evaluaciones concurrentes (de proceso) no sistemáticas. 
Por otra parte, los documentos analizados hacen mención a las cooperativas en el marco del análisis de los insumos monetarios, los productos y los resultados de las políticas sectoriales y de reconocimiento político que las tienen como beneficiarias, así como de las políticas sociales que incorporan la forma cooperativa como instrumento de organización. No presentan datos sobre el aporte económico y social o el desempeño de las cooperativas en su papel de promotoras (recursos movilizados, efectividad y eficiencia en la gestión de estos recursos, calidad de los productos y resultados, innovación, etc.). Tampoco ofrecen información sobre su desempeño como forma de organización para la inclusión social (prácticas de gestión y organización de la población beneficiaria en relación con los principios cooperativos, calidad del empleo creado, nivel de ingreso de los asociados, etc.), lo que refuerza el rol instrumental del cooperativismo en este tipo de políticas públicas.

Tabla 2

Ejemplos de indicadores utilizados por los organismos públicos nacionales

\begin{tabular}{|c|c|c|c|c|}
\hline $\begin{array}{l}\text { Tipo de } \\
\text { participación }\end{array}$ & $\begin{array}{l}\text { Tipo de política } \\
\text { pública }\end{array}$ & Componente & Indicador & $\begin{array}{l}\text { Tipo de } \\
\text { indicador }\end{array}$ \\
\hline Todas & Todas & Insumos & Porcentaje de dinero ejecutado & Cuantitativo \\
\hline Todas & Todas & Insumos & Porcentaje de dinero devengado & Cuantitativo \\
\hline Instrumental & Política social & Acciones & $\begin{array}{l}\text { Temas abordados en las capacitaciones de la pobla- } \\
\text { ción beneficiaria }\end{array}$ & Cualitativo \\
\hline Instrumental & Política social & Acciones & $\begin{array}{l}\text { Cantidad de convenios firmados con entes ejecu- } \\
\text { tores }\end{array}$ & Cuantitativo \\
\hline Instrumental & Política social & Acciones & $\begin{array}{l}\text { Elaboración de los contenidos del taller denomina- } \\
\text { do "Trayectoria laboral y formación ciudadana" }\end{array}$ & Cualitativo \\
\hline Instrumental & $\begin{array}{l}\text { Reconocimiento } \\
\text { político }\end{array}$ & Productos ${ }^{*}$ & $\begin{array}{l}\text { Cantidad de personas inscriptas en el régimen de } \\
\text { monotributo social }\end{array}$ & Cuantitativo \\
\hline Promotora & Política social & Productos & $\begin{array}{l}\text { Cantidad de organizaciones intermediarias capaci- } \\
\text { tadas en microcréditos }\end{array}$ & Cuantitativo \\
\hline Instrumental & Política social & Resultados & $\begin{array}{l}\text { Cantidad de personas capacitadas en formación } \\
\text { laboral }\end{array}$ & Cuantitativo \\
\hline Instrumental & Política social & Resultados & Tipo de obras realizadas & Cualitativo \\
\hline Instrumental & Política social & Resultados & Cantidad de personas beneficiarias del programa & Cuantitativo \\
\hline Instrumental & Política sectorial & Resultados & $\begin{array}{l}\text { Cantidad de viviendas terminadas, construidas con } \\
\text { cooperativas de trabajo }\end{array}$ & Cuantitativo \\
\hline Instrumental & Política sectorial & Resultados & $\begin{array}{l}\text { Cantidad de obras de expansión de agua potable y } \\
\text { cloaca con cooperativas de trabajo }\end{array}$ & Cuantitativo \\
\hline Instrumental & Política social & Producto & Cooperativas creadas & Cuantitativo \\
\hline Instrumental & Política social & Producto & $\begin{array}{l}\text { Unidades productivas que recibieron asistencia } \\
\text { financiera }\end{array}$ & Cuantitativo \\
\hline Beneficiarias & $\begin{array}{l}\text { Reconocimiento } \\
\text { político }\end{array}$ & Producto & Cantidad de mutuales y cooperativas controladas & Cuantitativo \\
\hline Beneficiarias & $\begin{array}{l}\text { Reconocimiento } \\
\text { político }\end{array}$ & Producto & Creación de nuevas cooperativas & Cuantitativo \\
\hline Beneficiarias & $\begin{array}{l}\text { Reconocimiento } \\
\text { político }\end{array}$ & Resultados & Cantidad de asociados a cooperativas capacitados & Cuantitativo \\
\hline Beneficiarias & Política sectorial & Producto & $\begin{array}{l}\text { Cantidad de cooperativas y mutuales que recibie- } \\
\text { ron asistencia financiera }\end{array}$ & cuantitativo \\
\hline
\end{tabular}

Nota. Elaboración propia con base en informes de organismos públicos nacionales. Este indicador refleja un producto dado que el resultado de esta acción lo refleja; por ejemplo, el número de trabajadores formalizados a través del régimen de monotributo. 
En las estadísticas públicas oficiales - censos de población, encuestas empresariales o del mercado de trabajo- la escasa diferenciación entre empresas de propiedad colectiva y no colectiva dificulta la creación de indicadores de seguimiento y la comparación en términos de datos cuantitativos y cualitativos. Solo el censo agropecuario introduce una variable vinculada a las cooperativas como forma asociativa y canal de comercialización de las explotaciones agropecuarias. Esta información, sin embargo, solo alcanza a describir una estrategia de los productores, pero nada dice sobre los productos y los resultados de la relación entre las cooperativas agropecuarias y los productores asociados.

La relevancia económica y social de las cooperativas no solo se expresa en su participación en las políticas sociales y con enfoque territorial - bien sea como promotoras o bien como instrumentos de organización - sino también, y principalmente, en el desarrollo de su actividad económica e institucional. Es en estos ámbitos de acción que las cooperativas generan productos y resultados que impactan en el ingreso (o ahorro) de sus asociados, así como en el crecimiento a largo plazo y la resiliencia de sus territorios.

El Instituto Nacional de Asociativismo y Economía Social (Inaes) es un organismo nacional descentralizado encargado de la reglamentación, la fiscalización y la promoción de las cooperativas y mutuales. En los últimos años, este instituto ha mejorado la calidad de la información disponible sobre el sector cooperativo y mutual. No obstante, aún existen importantes limitaciones para caracterizar adecuadamente la estructura y la participación económica y social de estas entidades en la sociedad (Acosta, Levin y Verbeke, 2013). Entre el 2005 y el 2007 este organismo realizó el "Reempadronamiento Nacional Obligatorio de Cooperativas y Mutuales", y el "Censo Económico Sectorial de Cooperativas y Mutuales" (Inaes, 2008). Estos instrumentos los consideró el organismo como "el punto de partida de un proceso que atiende a la necesidad de contar con una base de datos estadística integral, para el conocimiento de la realidad de las entidades en todo el territorio nacional" (Inaes, 2008, p. 9). Sin embargo, la información generada a partir de estos instrumentos carece de uniformidad al tomar como criterio el sector de actividad económica en lugar del objeto social de las cooperativas, y no ha sido planteada como el inicio de un proceso de recolección sistemática de datos (Acosta et al., 2013).

A diferencia de otras fuentes de datos, el documento generado por el Inaes destaca el papel de las cooperativas como promotoras de mejores condiciones económicas para sus asociados y su comunidad en general. Sus datos abordan el alcance del sector, sus recursos institucionales, los productos y los resultados de su actividad económica, así como su situación financiera-patrimonial (véase la tabla 3).

Tabla 3

Indicadores utilizados en el "Censo Económico Sectorial de Cooperativas y Mutuales" del Inaes

\begin{tabular}{ll}
\hline \multicolumn{1}{c}{ Dimensiones } & \multicolumn{1}{c}{ Indicadores } \\
\hline Alcance & $\begin{array}{l}\text { Número de asociados por provincia. } \\
\text { Número de entidades por sector de actividad, provincia, antigüedad, objeto social, sector de } \\
\text { actividad económica, etc. }\end{array}$ \\
\hline Recursos institucionales & Número de cooperativas que participan en entidades de segundo y tercer grado. \\
& Número de cooperativas según canales de comunicación con los asociados y la comunidad. \\
& Número de autoridades según antigüedad, género, nivel de educación por región geográfica. \\
& Número de entidades con gerencias según sector de actividad. \\
& Número de gerentes según nivel y tipo de educación, género, antigüedad. \\
& Personal ocupado por sector de actividad. \\
& Número de entidades con equipamiento informático y acceso a Internet. \\
& Número de capacitaciones, horas de capacitación y cantidad de participantes por sector de \\
actividad.
\end{tabular}


Dimensiones

Productos y resultados de la actividad económica

Indicadores

Número de entidades y volumen de productos agropecuarios y agroindustriales procesados o comercializados.

Número de entidades según capacidad de almacenaje.

Número de entidades según tipo de servicios prestados y actividades de I\&D.

Monto otorgado en préstamo por las cooperativas de crédito según destino y región.

Tipo de capacidad instalada de las cooperativas en el sector salud.

Cobertura del servicio de energía eléctrica por región de las cooperativas de servicios públicos.

Otros indicadores específicos de cada sector de actividad.

\begin{tabular}{ll}
\hline $\begin{array}{l}\text { Situación financiera- } \\
\text { patrimonial }\end{array}$ & Valor total del patrimonio neto, ingresos y egresos según sector de actividad y región. \\
& Valor total de los activos y pasivos según sector de actividad en quintiles. \\
& $\begin{array}{l}\text { Monto total de ingresos, egresos, subsidios, inversión y endeudamiento según sector } \\
\text { de actividad en quintiles. }\end{array}$ \\
\hline
\end{tabular}

Nota. Elaboración propia con base en Inaes (2008).

Algunas de estas medidas - como, por ejemplo, el valor de los activos, los ingresos totales, el número de asociados y los puestos de trabajo- se utilizan a nivel internacional con el fin de medir la participación y el peso de las cooperativas en la economía (Altman, 2017; Artis et al., 2015). No obstante, la información generada por el Inaes, en algunos casos, no discrimina entre cooperativas y mutuales $y$, en general, no expresa el alcance y la calidad de los resultados y productos que brindan las entidades ni su incidencia en el bienestar de los asociados. Estas condiciones imposibilitan evaluar la participación económica de las cooperativas en consideración a su tamaño, su ubicación geográfica y su impacto en el territorio. Además, no contempla en su análisis otras contribuciones sociales que realiza el cooperativismo como, por ejemplo, el compromiso cívico, la actividad política, la innovación social, etc. (Artis et al., 2015).

Por último, el informe del Inaes dedica una sección especial a las cooperativas de trabajo creadas en el marco de políticas sociales, en la cual se menciona la cantidad de entidades según el programa social que le da origen, su sector de actividad económica, su ubicación geográfica y la cantidad de asociados.

En cuanto a las fuentes de información privadas, algunas federaciones de cooperativas brindan datos agregados sobre el desempeño económico y financiero de sus organizaciones asociadas, para lo cual recurren a variables como: volumen de producción, nivel y alcance geográfico de la demanda, monto de inversión, etc. Sin embargo, esta información, centrada en los productos que generan las entidades, raramente se encuentra actualizada, no es sistemática ni homogénea y no expresa el impacto de la relación cooperativa-asociado.

Otra fuente de información privada promovida por la Alianza Cooperativa Internacional (ACI) y con una creciente aceptación entre las cooperativas es el balance social cooperativo (BSC). Este documento lo elaboran las cooperativas según sus preferencias y criterios ${ }^{5}$ con el objetivo de brindar información a sus actores de interés sobre las acciones desarrolladas y su correspondencia con los valores y los principios que las rigen. Este enfoque anclado en la realidad local y específica de cada entidad se diferencia de otras medidas que permiten la comparación con el resto de la economía y otras entidades (Artis et al., 2015).

El BSC ofrece información sistemática y comparable a lo largo del tiempo sobre las acciones que realiza cada cooperativa, los insumos que aporta para la realización de actividades propias o de otras organizaciones y los productos y resultados que obtienen en el desempeño de su actividad productiva, social e institucional. En este marco, el registro

5 Si bien cada cooperativa puede elegir el modelo de Bsc y las dimensiones y variables que lo componen, la ACI ofrece una certificación en Responsabilidad Social Cooperativa a aquellas entidades que lo soliciten: "En virtud de reconocer que los resultados del informe de BSC, se ajustan al modelo sugerido por ACI-Américas y se enmarcan en el análisis y ponderación del cumplimiento de los principios cooperativos" (ACI, 2009). 
de los recursos, los productos y los resultados derivados de la acción cooperativa y su valorización económica permitirían medir la contribución económica que realizan estas entidades a la sociedad y su transformación en bienes y servicios. Un ejemplo de esta medición surge al evaluar los recursos, los productos, los resultados y los impactos derivados de las capacitaciones que ofrecen las cooperativas a sus asociados bajo el principio "Educación, formación e información". Las horas/hombre dedicadas a su organización y los salones destinados a esta actividad forman parte de los insumos suministrados por la cooperativa, el número de encuentros de capacitación y el material provisto a los participantes son productos, mientras que el número de personas alcanzadas por esta actividad, por ejemplo, forman parte del resultado. El impacto de esta actividad dependerá de la temática de la capacitación, pero podría incidir en el nivel de participación de los asociados en la gestión de la cooperativa, en la productividad de los productores asociados, en un aumento del volumen producido, etc. Si los insumos aplicados y los impactos alcanzados se valorizan, al recurrir, por ejemplo, a su costo de oportunidad o de reemplazo, sería posible contar con una estimación monetaria del aporte y el impacto de estas entidades en el marco de las políticas públicas que las involucran y en la comunidad en general.

A pesar de sus potencialidades para visibilizar la relevancia económica y la legitimidad social del sector, así como sus aportes en el marco de políticas públicas, el BSC presenta algunas limitaciones (Fernández-Lorenzo, Geba, Montes y Schaposnik, 1998):

- Existen diversos modelos y criterios para elaborar un balance social, por lo cual la información que brindan estos documentos no siempre es comparable y, por tanto, no es posible agregar en todos los casos la información por tipo de entidad o sector de actividad.

- El Bsc no es aplicado por todas las cooperativas, ya que aun cuando existe una tradición contable en el sector para informar sobre la gestión de los recursos de la entidad y sus resultados económicos, las entidades, por lo general, no poseen sistemas de información integral que den cuenta de sus finalidades sociales.

- El BSC se centra en presentar los recursos, los productos y los resultados generados por las entidades y propone evaluar el impacto de las entidades en la calidad de vida de los asociados, de los trabajadores y de la comunidad mediante la cuenta Valor Agregado Cooperativo Visibilizado e Invisibilizado. Sin embargo, el cálculo de esta cuenta no es común a todos los BSC, pues requiere que las entidades cuenten con registros sistematizados de sus acciones y tomen decisiones metodológicas para su elaboración.

\section{Conclusiones}

En Argentina, como en otros países de América Latina y Europa, el Estado nacional ha formulado políticas públicas basadas en la articulación Estado-mercado-sociedad, y ha planteado espacios más o menos estructurados y formalizados de interacción entre la administración pública y nuevos actores sociales. En el periodo 2003-2015, las cooperativas han participado en las políticas públicas nacionales de diferentes formas: como beneficiarias, como instrumentos de organización o como promotoras de estas intervenciones; así como con el aporte y la gestión de recursos, la generación de productos y resultados, o con el logro de impactos en sus territorios.

Estas contribuciones, sin embargo, no se encuentran registradas en los sistemas de rendición física y financiera que posee el Estado nacional, ni en las estadísticas públicas oficiales. Los sistemas de rendición del Estado se centran en los recursos monetarios aportados por el Gobierno nacional, y en los productos y resultados derivados de las políticas públicas, de modo que se limita a registrar la cantidad y no la calidad de estos elementos. Tampoco registran el desempeño de las cooperativas en su función, bien sea como instrumento de organización de la población beneficiaria (nivel de transparencia y participación, prácticas de gestión, etc.), o bien como agente promotor de una política pública (efectividad en la gestión de los recursos públicos, desarrollo de productos innovadores, etc.), incluso en relación con otras formas de organización tales como asociaciones civiles y municipios. La ausencia de este tipo de información limita la toma de decisiones de las autoridades públicas respecto a cambios en la formulación y la gestión de las políticas públicas, pero además condiciona el reconocimiento del rol de las cooperativas tanto por parte del Estado como de la sociedad.

Por otra parte, la información registrada por el Inaes en el censo de cooperativas y mutuales 
- aunque limitada en algunos aspectos- ofrece datos sumamente relevantes para dimensionar el alcance del sector y su participación en la economía nacional, tales como activos, pasivos, resultados, etc. Sin embargo, su alto nivel de agregación y la falta de continuidad en su registro condicionan la posibilidad de evaluar el impacto económico de estas entidades por objeto social y a nivel provincial. Estas condiciones dificultan el acceso a canales de diálogo con el Estado y otros actores privados, así como a recursos públicos con foco en las cooperativas como motor de crecimiento económico y desarrollo comunitario.

Por último, el BSC representa una oportunidad para establecer una metodología de recopilación, estimación y valorización de los recursos, los productos y los resultados generados por cada cooperativa, tanto en el marco de su actividad económica como de las políticas públicas en las que participa. Aunque su difusión requiere la movilización de múltiples recursos para adaptar el instrumento a cada entidad sin perder comparabilidad, adecuar los registros administrativos de las cooperativas y formar recursos humanos de las entidades a fin de diseñar y elaborar el BSC fortalecería el objetivo del BsC y lo transformaría en un instrumento de medición armonizado que permita evaluar la contribución de las cooperativas a la economía y a la sociedad en general.

\section{Referencias}

ACI. (2009). ACI-Américas nominada en Innovación por la Cámara de Comercio de Costa Rica. Recuperado de: www.aciamericas.coop/ACI-Americas-nominada-en?tema $=60 \& \mathrm{nb}=80$.

Acosta, M. C., Levin, A., y Verbeke G. E. (2013). El sector cooperativo en Argentina en la última década. Cooperativismo \& Desarrollo, 21(102), 27-39.

Altman, M. (2017). The importance of co-operatives to the New Zealand economy: constructing a co-operative economy. Recuperado de: https://ssrn.com/ abstract $=2990326$.

Amyot, S., Downing, R., y Tremblay, C. (2010). Public policy for the social economy: building a people-centered economy in Canada. Victoria: Canadian Social Economy Hub at the University of Victoria.

Artis, A., Bouchard, M. J., y Rousseliere, D. (2015). Léconomie sociale compte-t-elle? Comment la compte-ton? Représentations de l'économie sociale à travers les indicateurs statistiques (Docuemento de trabajo, CIRIEC 2015/02).
Casanova, F. (2004). Desarrollo local, tejidos productivos y formación. Abordajes alternativos para la formación $y$ el trabajo de los jóvenes. Montevideo: Cinterfor/ OIT.

Castelao Caruana, M.E. (2013). La economía social y solidaria en las políticas públicas nacionales y su incidencia en el territorio: alcance y perspectivas (tesis de doctorado). Universidad de Buenos Aires, Buenos Aires, Argentina.

Chaves, R. (2012). Las políticas públicas y las cooperativas. Ekonomiaz, 79(1 ${ }^{\text {er }}$ cuatrimestre), 168-199.

Chaves, R., y Demoustier, D. (2013). Introduction. En R. Chaves y D. Demoustier (Comp.), The emergence of the social economy in public policies. An international analysis (pp. 11-18). Bruselas: Peter Lang.

Cortés, M. (2008). Movimientos sociales y Estado en Argentina: entre la autonomía y la institucionalidad. Buenos Aires: Clacso.

Fernández-Lorenzo, L., Geba, N., Montes, V., y Schaposnik, R. (1998). Balance Social Cooperativo Integral. Un modelo argentino basado en la identidad cooperativa. La Plata: UNLP.

Gertler, P., Martínez, S., Premand, P., Rawlings, L., y Vermeersch, C. (2017). La evaluación de impacto en la práctica. Washington, D. C.: Banco Interamericano de Desarrollo y Banco Mundial.

Guanziroli, C. E., Buainain, A. M., y de Sousa-Filho, H. M. (2007). Metodología de evaluación del impacto y de los resultados de los proyectos de cooperación técnica. Brasilia: Instituto Interamericano de Cooperación para la Agricultura.

Instituto Movilizador de Fondos Cooperativos-IMFC. (2008). Propuesta para construir un país con más democracia y equidad distributiva. Buenos Aires: IMFC.

Inaes. (2008). Las cooperativas y las mutuales en la República Argentina. Reempadronamiento nacional y Censo económico sectorial de cooperativas y mutuales. Buenos Aires: Inaes.

Klein, J.-L., Fontan, J.-M., Saucier, C., Trembaly, D. G., Tremblay, P. A., y Simard, M. (2010). Les conditions de réussite des initiatives locales de lutte contre la pauvreté et l'exclusion qui mobilisent des ressources de l'économie sociale. Québec: Crises.

Ley nacional 20.337/1973 de 2 de 1973. Ley de Cooperativas. Nuevo Regimen. Mayo 15 de 1973. Boletín Oficial n. ${ }^{\circ}$ 22.666. [Argentina].

Ley nacional 25.865/2003 de 17 de diciembre. IVA-Monotributo. Regimen Legal-Modificaciones. Enero 19 de 2004. Boletín Oficial n. ${ }^{\circ}$ 30.320. [Argentina].

Ley nacional 26.117/2006 de 28 de junio. Promoción del microcrédito para el desarrollo de la economía social. Julio 21 de 2006. Boletín Oficial n. 30.952. [Argentina]. 
Organización Internacional del Trabajo-orT. (2011). Economía social y solidaria: nuestro camino común hacia el trabajo decente. Turin: Centro Interancional de Formación de la orT.

Pestoff, V. (2006). Citizens and co-production of welfare services. Public Management Review, 8(4), 503-519.

Pochmann, M. (2007). Posibilidades y límites de la economía solidaria latinoamericana. En J. L. Coraggio, La economía social desde la periferia. Contribuciones latinoamericanas (pp. 225-240). Buenos Aires: UNGS-Altamira.

Resolución spsydh 1.375/2004 de abril de 2004 [Secretaría de Políticas Sociales y Desarrollo Humano]. Creación del Plan Nacional de Desarrollo Local y Economía Social "Manos a la Obra".

Ropert, M. A. (2009). Evolución de la política de desarrollo económico territorial en Chile: principales iniciativas. Santiago de Chile: Rimisp.

Rouillard, C. (2006). Les partenariats public-privé et la reconfiguration de la gouvernance: réflexion sur la construction d'un État enthropique. Les Cahiers, 3(1), 33-37.

Singer, P. (2007). Economía solidaria. Un modo de producción y distribución. En J. L. Coraggio, La economía social desde la periferia. Contribuciones latinoamericanas (pp. 59-78). Buenos Aires: UNGS-Altamira.
Spear, R. (2000). Organismos de apoyo. En R. Chaves et al., economía social y empleo en la Unión Europea (pp. 85-133) Madrid: Ciriec-España.

Subirats, J., Knoepfel, P., Larrue, C., y Varone, F. (2008). Análisis y gestión de políticas públicas. Barcelona: Ariel.

Tremblay, C. (2010). Public policy trends and instruments supporting the social economy: international experiences. Victoria: Canadian Social Economy Hub.

Vaillancourt, Y. (2009). Social economy in the co-construction of public policy. Annals of public and cooperative economics, 80(2), 275-313.

Vinocur, P., y Halperin, L. (2004). Pobreza y políticas sociales en Argentina de los años noventa. Santiago de Chile: Cepal. Recuperado de: repositorio.cepal.org/ bitstream/handle/11362/6074/1/S044231_es.pdf.

Vuotto, M. (2014). La economía social y las cooperativas en la Argentina. Voces en el Fénix, (38), 46-53. Recuperado de: www.vocesenelfenix.com/content/acerca-del-cooperativismo-de-trabajo-en-la-argentina 